\title{
Irène ALBERS, Claude Simon. Moments photographiques
}

\section{Antonin Wiser}

\section{Q OpenEdition}

1 Journals

Édition électronique

URL : https://journals.openedition.org/ccs/620

DOI : $10.4000 /$ ccs.620

ISSN : 2558-782X

Éditeur :

Presses universitaires de Rennes, Association des lecteurs de Claude Simon

\section{Édition imprimée}

Date de publication : 30 novembre 2008

Pagination : 155-157

ISBN : 9782354120351

ISSN : 1774-9425

\section{Référence électronique}

Antonin Wiser, «Irène ALBERS, Claude Simon. Moments photographiques », Cahiers Claude Simon [En ligne], 4 | 2008, mis en ligne le 21 septembre 2017, consulté le 26 avril 2022. URL : http:// journals.openedition.org/ccs/620; DOI : https://doi.org/10.4000/ccs.620

\section{Ce document a été généré automatiquement le 26 avril 2022}

Cahiers Claude Simon 


\title{
Irène ALBERS, Claude Simon. Moments photographiques
}

\author{
Antonin Wiser
}

\section{RÉFÉRENCE}

Irène ALBERS, Claude Simon. Moments photographiques, Presses Universitaires du Septentrion, Villeneuve d'Ascq, 2007, 269 p., traduit de l'allemand par L. Cassagnau.

Dans cette riche étude, Irène Albers, professeur à l'université libre de Berlin, se propose d'explorer les relations multiples qu'entretient l'œuvre de claude simon avec la photographie. Ces relations, placées sous le signe d'une esthétique du choc, se trouvent caractérisées par «le fait que Simon voit dans la photographie et la guerre les médias d'une vision nouvelle, d'une vision qui est esthétiquement séduisante parce qu'elle brise les ordres de la langue et en réclame une nouvelle. Ainsi la réflexion sur les médias et sur la langue, la théorie de la photographie et du roman sont indissociablement liées » (p. 20). Partant de cette hypothèse, c'est donc à une analyse médiologique de l'œuvre simonienne que l'auteur se livre ici.

2 Albers esquisse tout d'abord un tableau de la réception de la technique photographique dans les milieux littéraires dans la première moitié du $\mathrm{XX}^{\mathrm{e}}$ siècle, pour mieux situer l'originalité de la position simonienne. Convoquant tour à tour Proust, Benjamin et Kracauer, Albers met en évidence tout à la fois leur fascination et leur méfiance à l'égard de la photographie. Ce qui est en jeu à leurs yeux, c'est un rapport à la mémoire profondément transformé par la fixation d'un instant fugitif sur la pellicule : là où le souvenir, certes moins précis, conserve l'image du passé au sein d'un contexte qui la maintient vivante, la photographie découpe un instantané dans le continuum temporel pour l'en isoler et produit ainsi un "effet de choc». Alors que l'attitude de ces trois écrivains reste ambivalente à l'égard de cet « effet de choc », Simon le met pour sa part positivement au service de son esthétique et de sa pratique romanesque. Ainsi, «la photographie peut se faire le modèle adéquat d'un souvenir d'abord traumatique» ( $p$. 
49) qui hante toute l'œuvre simonienne. Ce modèle offre dès lors - c'est la thèse que développe Albers dans son ouvrage - une clef de lecture de la poétique de Simon.

La lecture médiologique proposée par Albers suit plusieurs axes. Le premier, thématique, concerne l'usage métaphorique de la photographie, notamment dans $L a$ Corde raide, Le Vent et La Route des Flandres. Le cliché photographique y apparait non seulement comme métaphore de la perception et du souvenir, mais il permet également à Simon de décrire de manière plus générale une certaine structure de la temporalité vécue: le caractère discret et fixe des images répond en effet à une expérience du temps et du monde marquée par des solutions de continuité et l'éclatement de l'unité du sujet. L'«Achille immobile à grand pas» de La Bataille de Pharsale se laisse ainsi illustrer par ces paradoxales chronophotographies qui représentent le mouvement d'un homme courant par une succession d'instantanés figés. Par diverses stratégies textuelles - dont l'emploi du participe présent est certainement la plus massive -, l'écriture de Simon semble se faire elle-même chronophotographique et libère alors "l'énergie et l'expérience de "violence" comprimées dans les images instantanées et les [déploie] comme dynamique textuelle » (p. 134).

4 Après un regard sur la production photographique proprement dite de Simon (Album d'un amateur et Photographies), Albers se penche sur sa pratique et sa théorisation de la " description dynamique », pour mettre en évidence ce qu'elles doivent à une réflexion sur la photographie. La fonction des mots-carrefours comme vecteurs dynamiques entre deux descriptions semble tout d'abord travailler à reconstruire des connexions que l'image, isolée et décontextualisée, brise nécessairement. Toutefois, l'analyse d'Albers met en évidence que les sauts selon lesquels s'opèrent chez Simon ces reconnections, les «shift » d'un personnage, d'un temps, d'un lieu à l'autre, supposent une esthétique qui a d'abord assimilé le caractère discret et discontinu des images photographiques.

5 L'étude s'achève par l'analyse détaillée du travail scriptural à partir de ces stimuli iconographiques que sont les photos de famille dans L'Herbe et les cartes postales dans Histoire. Albers suggère qu'il ne s'agit dans les deux cas pas tant de la représentation de l'image par le texte que de «la transformation des photographies en textes, d'un problème de médias du souvenir, du rapport des différents médias - photographie et langue - à ce qui a été oublié, passé sous silence, refoulé, au non-dit et au nonracontable de l'histoire (familiale)» (p. 191). L'image provoque littéralement le texte par son mutisme propre : le visible est lu en fonction d'un invisible hors champ, ainsi que le notait déjà l'auteur à propos des clichés pris par Simon. Il en va exactement de même pour la photographie de l'atelier qui fait l'objet du chapitre central d'Histoire. Les «qualités médiales» de la photographie -son isolement d'un continuum temporel qu'elle invite à reconstruire sous hypothèse, mais également le «bougé » qui enregistre en elle l'espacement du temps -enclenchent une description dynamique de telle sorte que le cliché se trouve "en quelque sorte "développé" une deuxième fois par cette technique de lecture» (p. 222). À ce point, la photographie perd son caractère de document pour devenir le lieu d'inscription des «traces douteuses» d'un passé incertain que l'écriture vient investir pour suppléer ce qui en l'image fait défaut. Dès lors l'insistance sur la textualité ne relève pas d'abord d'un exercice autoréférentiel sur la langue mais doit plutôt être « référée aux essais infructueux d'éclairer le phénomène de la référentialité sous forme de trace » (p. 245). 
6 Claude Simon, moments photographiques constitue une contribution extrêmement stimulante à l'étude de l'œuvre simonienne, offrant des éclairages originaux tant sur les dimensions poétiques que thématiques de celle-ci. Par ailleurs, et ce n'est pas la moindre de ses qualités, l'ouvrage d'Albers illustre le potentiel considérable de la Medienanalyse - encore relativement peu pratiquée en France - pour l'analyse littéraire. 